\title{
Flow Oscillations and Meniscus Fluctuations in a Funnel-Type Water Mold Model
}

\begin{abstract}
YOUNG JIN JEON, HYUNG JIN SUNG, and SANGMIN LEE
Transient flows in a funnel-type continuous casting process model were studied experimentally to investigate the flow oscillations inside the mold and the meniscus fluctuations. A full-scale water model was used with dimensions of $2000 \mathrm{~mm}$ (length) $\times 1350 \mathrm{~mm}$ (width) $\times 100 \mathrm{~mm}$ (thickness). Particle image velocimetry (PIV) was employed to measure the flow oscillations. To minimize high shear flow errors near the submerged entry nozzle (SEN) exit, the window deformation technique was adopted. The meniscus levels were extracted by edge-detection image processing. Three types of SEN and two funnel thicknesses $(180 \mathrm{~mm}$ and $220 \mathrm{~mm})$ were tested to examine the flow characteristics under five flow rates $\left(10,20,30,40\right.$, and $\left.50 \mathrm{~m}^{3} / \mathrm{h}\right)$. The vortex generation mechanism inside the mold was analyzed across the various mold conditions studied.
\end{abstract}

DOI: $10.1007 / \mathrm{s} 11663-009-9318-\mathrm{z}$

(C) The Minerals, Metals \& Materials Society and ASM International 2009

\section{INTRODUCTION}

DURING the last 30 years, many studies have examined continuous casting processes based on a near-net-shape casting concept with the aim of reducing costs and improving productivity. Recently, thin slab casting has been used widely because of its high casting extraction speed and rolling efficiency. In such processes, however, the submerged entry nozzle (SEN) that supplies molten steel to the mold cannot be smaller than a certain size because of the high extraction speed. To overcome this limitation, the upper center region of the mold should be widened to form a funnel shape. However, this modification can cause unstable flow features at high flow rates, which include flow oscillations inside the mold and meniscus fluctuations. These unstable features can have a significant negative impact on the final product quality. To reduce such flow oscillations, water-model analysis can be used to identify an optimal SEN design. Previous attempts to achieve flow stabilization have devoted considerable attention to modeling the inner flow and the meniscus quantitatively.

In general, the flow in a continuous casting process has self-sustaining oscillations. These flow oscillations have been investigated through many numerical simulations and experimental measurements. Honeyands and Herbertson $^{[1]}$ used both a commercial simulation tool and a water model to examine such oscillations. They showed that the observed variations in the meniscus are related closely to self-sustained oscillations of the jets exiting from the SEN. Subsequently, Gebert et al. ${ }^{[2]}$ predicted the relation between the inner flow oscillations

YOUNG JIN JEON, PhD Candidate, and HYUNG JIN SUNG, Professor, are with the Department of Mechanical Engineering, KAIST, 373-1 Guseong-dong, Yuseong-gu, Daejeon 305-701, Korea. Contact e-mail: hjsung@kaist.ac.kr SANGMIN LEE, Principal Researcher, is with POSCO, PO Box 35, Pohang, Gyeongbuk 790-785, Korea.

Manuscript submitted May 28, 2009.

Article published online November 17, 2009. and the meniscus fluctuations using a two-dimensional numerical model. However, they did not compare their findings with experimental data. In terms of experimental studies, Gupta and Lahiri ${ }^{[3]}$ measured transient asymmetric flows in a water model and provided qualitative information on self-sustained flow oscillations in the mold. Lawson and Davidson ${ }^{[4]}$ observed the flow oscillations by varying the nozzle diameter as well as the mold width and thickness. They determined the conditions that gave stable cross flows.

In addition to flow oscillations, vortex generation inside the mold and the corresponding meniscus fluctuations are also important to the final product quality. An influx of mold powders from the meniscus elicits product cracking. The vortex formation mechanism is basically the same as the "bathtub vortex" described by Gebhard et al. ${ }^{[5]}$ and Li et al. ${ }^{[6]}$ The latter group showed numerically that an eccentric SEN causes vortex generation at the side of the SEN. In the experimental results of Li and Tsukihashi, ${ }^{[7]}$ several vortices were observed at the side of the SEN. Recently, in a system with a noneccentric SEN, Torres-Alonso et al. ${ }^{[8]}$ observed similar vortices in numerical and experimental tests in a funnel mold.

Complicated flow phenomena inside a mold mainly originate from the high extraction speed at the SEN exit. Lamant et al. ${ }^{[9]}$ showed that the high shear flows at the meniscus associated with the influx of mold powders may lead to product cracking. Iguchi et al. ${ }^{[10]}$ found in their experiments that the Kelvin-Helmholtz instability induced by high flow rates at the meniscus can lead to powder entrapment. These results established that high meniscus velocities should be avoided. In contrast to the wealth of data available for parallel molds, studies of funnel-shaped molds are relatively scarce. Nam et al..$^{[1]}$ simulated the mold flow features with heat transfer and solidification and analyzed the effect of using a funnelshaped mold. Torres-Alonso et al. ${ }^{[8]}$ simulated the flow patterns numerically in a funnel mold and examined 
such flows experimentally using particle image velocimetry (PIV). In their work, however, the test section was limited to the small region close to the SEN meniscus. Detailed flow measurements are needed to elucidate the overall meniscus fluctuations and the oscillations inside the mold.

The objective of the present study was to investigate the flow oscillations and meniscus fluctuations in a funnel-type mold with various SEN models. To achieve this aim, we constructed a full-scale water model with a funnel-type mold. PIV measurements were taken in the entire region, which included the side walls, the SEN, and the meniscus, with planar velocity fields. In general, the mold flow had a high-velocity gradient, especially in the vicinity of the SEN exit and the meniscus. The window deformation technique was employed to obtain more accurate velocity data and to reduce the uncertainty of the PIV results. The image parity exchange method was applied to the side walls to obtain more accurate shear properties near the walls. In addition, the location of the meniscus in each PIV image was digitized by the gradient method based on edge-detection. Three types of SEN models were chosen with two funnel sizes and five flow rates. A quantitative statistical description was developed based on the oscillations and the fluctuating meniscus of the model.

\section{EXPERIMENTAL APPARATUS AND PROCEDURE}

\section{A. Full-Scale Water Model}

A full-scale funnel-type water model was constructed. As shown in Figure 1, the mold dimensions were $2000 \mathrm{~mm}$ (length) $\times 1350 \mathrm{~mm}$ (width W) $\times 100 \mathrm{~mm}$ (thickness T). The funnel-shaped upper part was widened

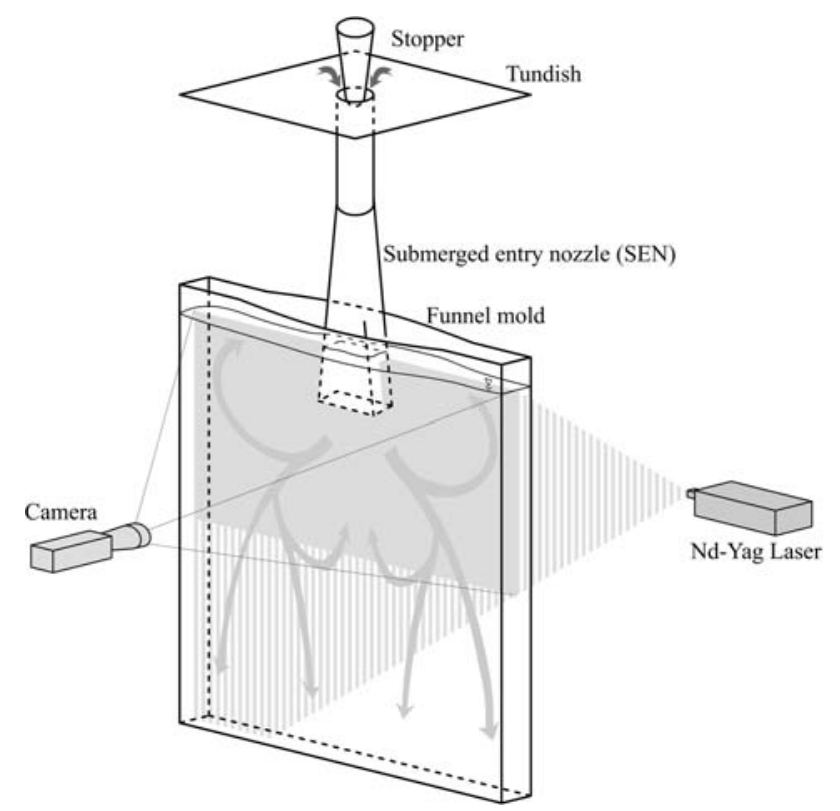

Fig. 1-Schematic of the experimental setup. slightly to install the SEN, and then the cross section of the mold was contracted gradually to the rectangular shape of the fixed bottom part. However, the side-slab part was kept constant (100 mm thickness, $0.074 \mathrm{~W}$ ) along the whole model. Silicon polymer was used to seal, and steel bars and bolts were used to prevent leakage caused by high hydrostatic pressure. Two funnel models were chosen - a narrow model of thickness $1.8 \mathrm{~T}$ $(180 \mathrm{~mm}, 0.1333 \mathrm{~W})$ and a broad model of thickness $2.2 \mathrm{~T}(220 \mathrm{~mm}, 0.1630 \mathrm{~W})$. The funnel shape was fabricated by heat treatment using a flat acryl plate $(20 \mathrm{~mm}$ thickness). The entire experiment was carried out with the temperature controlled to $20 \pm 1{ }^{\circ} \mathrm{C}$ to maintain constant flow properties.

A tundish tank mounted 850 to $950 \mathrm{~mm}$ above the mold supplied water with a constant head to the mold through the SEN; the flow head was controlled by a stopper in the tundish tank. Three SEN models were chosen for the flow measurements, as shown in Figure 2. Type I consisted of two exit nozzles oriented in a downward direction - a configuration that generally is used with a low casting speed. Type II had a pair of central nozzles and a pair of side nozzles to prevent "break out" by dispersion of the central flow. Type III was designed to distribute the flow with five nozzles. For all models, the total length was $1200 \mathrm{~mm}$, the inner diameter at the entrance of the SEN was $90 \mathrm{~mm}$ $(0.067 \mathrm{~W})$, and the corner between the SEN and the tundish was curved with a $30 \mathrm{~mm}$ radius.

The maximum sustainable extraction speed $(6.17 \mathrm{~m} /$ min) was selected as a maximum flow rate condition ( $\left.U=50 \mathrm{~m}^{3} / \mathrm{h}\right)$, and the following five flow rates were selected: $1.0 U, 0.8 U, 0.6 U, 0.4 U$, and $0.2 U$. When the flow rate was $1.0 U$, the Reynolds number based on the inner diameter and the mean velocity $(2.18 \mathrm{~m} / \mathrm{s})$ at the entrance of the SEN was $\mathrm{Re}=1.96 \times 10^{5}$. The PIV velocities were normalized by $U_{0}=0.1028 \mathrm{~m} / \mathrm{sec}$, which was based on the mean extraction speed under a flow rate of $1.0 \mathrm{U}$.

\section{B. PIV Measurement}

PIV techniques were employed to measure instantaneous velocity fields in the mold, as shown in Figure 1.

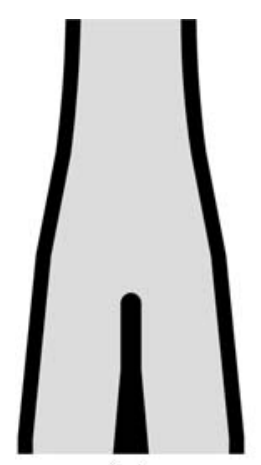

(a)

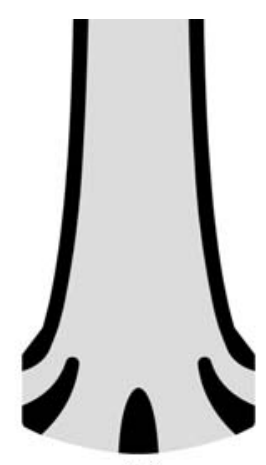

(b)

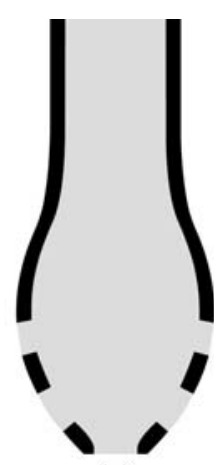

(c)
Fig. 2-Schematics of the submerged entry nozzles. (a) Type I, (b) Type II, and (c) Type III. 
A laser light sheet was produced by a double-pulsed Nd-Yag laser (New Wave Research, Fremont, CA) that delivered $200 \mathrm{~mJ}$ of energy per pulse at $532 \mathrm{~nm}$, which can cover both sides of the SEN $(1350 \mathrm{~mm} \times 1000 \mathrm{~mm})$ simultaneously. The laser sheet illumination was collimated by a concave cylindrical lens. Silver-coated hollow glass spheres with a mean diameter of $44 \mu \mathrm{m}$ were used as tracer particles in the flow fields. For the charge-coupled device (CCD) system, a Redlake ES4020 (Tallahassee, FL) camera capable of recording up to 15 frames per second with a resolution of $2048 \times 2048$ pix$\mathrm{els}^{2}$ was used to obtain the PIV images. Grayscale image pairs with $2048 \times 1280$ pixels $^{2}$, which have 256 intensity levels (8-bit), were captured at $5 \mathrm{~Hz}$, and the time interval between successive images was set at $d t=2.0$ to $4.0 \mathrm{~ms}$ because of the dynamic PIV range. For each flow condition, 5000 instantaneous PIV images were acquired.

As mentioned, a high flow rate at the SEN exit generates recirculation flows with high-velocity gradients. In such flows, the difference between the maximum and minimum velocity is large. In PIV image processing, the time interval $d t$ depends on the largest particle displacement, so the region around the exit of the SEN must be treated differently from the rest of the flow. Specifically, a small $d t$ was used for the region around the SEN exit and a larger $d t$ was used for the remainder of the system. Using a synthetic PIV test, Raffel et al. ${ }^{[12]}$ observed a large bias and random errors in high shear flows and large relative errors in slow flows in which particle displacements were small. These features originate from a loss-of-pair problem in the cross-correlation calculations in the PIV procedure. To compensate for the loss of particle pairs, Ashforth-frost et al., ${ }^{[13]}$ Huang et al..$^{[14]}$ and Jambunathan et al. ${ }^{[15]}$ developed a PIV algorithm that considers the flow pattern and distortion. In the window deformation algorithm, the cross-correlation between two interrogation windows is expressed as follows:

$$
\int_{y_{0}-w / 2}^{y_{0}+w / 2} \int_{x_{0}-w / 2}^{x_{0}+w / 2} f_{1}(x, y) \cdot f_{2}(x+\Delta x, y+\Delta y) d x d y
$$

where $w$ is the size of the square interrogation window, $\left(x_{0}, y_{0}\right)$ represents the center location of the first interrogation window, and $f_{1}$ and $f_{2}$ denote the image intensity fields expressed with the absolute coordinate $(x, y)$. The distributions of $\Delta x$ and $\Delta y$, which maximize Eq. [1], are what need to be determined. In particular, we consider the linear window deformation case, as shown in Figure 3. In this case, $\Delta x$ and $\Delta y$ are expressed as follows:

$$
\begin{aligned}
& \Delta x=\Delta x(x, y)=\Delta x\left(x_{0}, y_{0}\right)+\frac{\partial \Delta x}{\partial x}\left(x-x_{0}\right)+\frac{\partial \Delta x}{\partial y}\left(y-y_{0}\right) \\
& \Delta y=\Delta y(x, y)=\Delta y\left(x_{0}, y_{0}\right)+\frac{\partial \Delta y}{\partial x}\left(x-x_{0}\right)+\frac{\partial \Delta y}{\partial y}\left(y-y_{0}\right)
\end{aligned}
$$

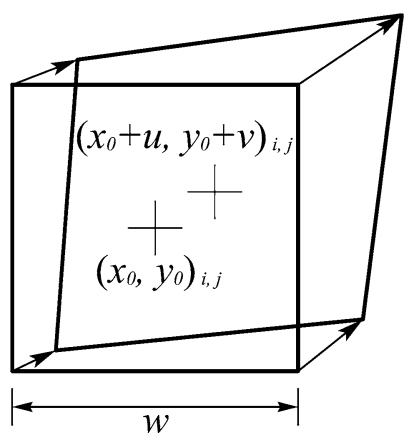

Fig. 3-Window deformation scheme.

As an interpolation scheme during the linear deformation process, a sinc function with a $6 \times 6$ kernel size was adopted to obtain the image data for the wall regions because of its good performance. ${ }^{[16]}$

$$
\begin{aligned}
f_{2}(x+\Delta x, y+\Delta y) \\
=\sum_{i=-2}^{+3} \sum_{j=-2}^{+3} f_{2}(x+i, y+j) \cdot \frac{\sin (\Delta x-i)}{\Delta x-i} \cdot \frac{\sin (\Delta y-j)}{\Delta y-j} .
\end{aligned}
$$

Furthermore, masking was applied to eliminate luminance and reflection from the side walls. To improve the gradients of the velocity vectors, the PIV images were expanded across the walls using the image parity exchange (IPX) introduced by Tsuei and Savas. ${ }^{[1]]}$ For the first step of the PIV process, an iterative multigrid algorithm ${ }^{[18]}$ was used, and a square interrogation window with a size of $w=128$ pixels was employed as a predictor. The final window size was set to $w=64$ pixels with 50 pct overlap, and five iterative calculations of a cross-correlation were applied with the window deformation algorithm.

\section{Detection of Meniscus Levels}

Measurement of the time-varying flow field is a general method to investigate the flow oscillations. Obtaining data on the cross flow around the funnel near the exit of the SEN is an essential aspect of measuring the oscillations. However, in the present PIV measurement setup, it was not possible to obtain velocity data in the vicinity of the SEN. Instead, we measured the meniscus levels, which were directly related to the cross flow around the funnel. An algorithm for determining the meniscus location was applied. ${ }^{[19]}$ Figure 4 shows a sample PIV image in which the meniscus can be detected easily. Law et al. ${ }^{[19]}$ directly obtained the meniscus locations on PIV images using the sudden change in intensity at the interface. This technique worked well for images such as Figure 4(a). In the present experiments, however, the meniscus was sometimes hidden by a shadow (Figure 4(b)) or obscured by bright diffuse reflections (Figure 4(c)). Such defects may cause the meniscus detection algorithms based on a sudden change in 
intensity to give unrealistic meniscus positions. To improve the reliability of the meniscus detection algorithm, the definite integral of the PIV image in the horizontal direction was calculated as follows:

$$
I(x, y)=\int_{x-d x / 2}^{x+d x / 2} f(x, y) d x
$$

where eight pixels are used for $d x$. To find the meniscus, the convolution function $c(\tau)$, which is shown in Figure 5(b), was introduced. The maximum position was obtained as the meniscus location by applying the convolution to $I(x, y)$,

$$
y_{\text {meniscus }}=\max \int_{-\infty}^{+\infty} c(\tau) I(x, y-\tau) d \tau
$$
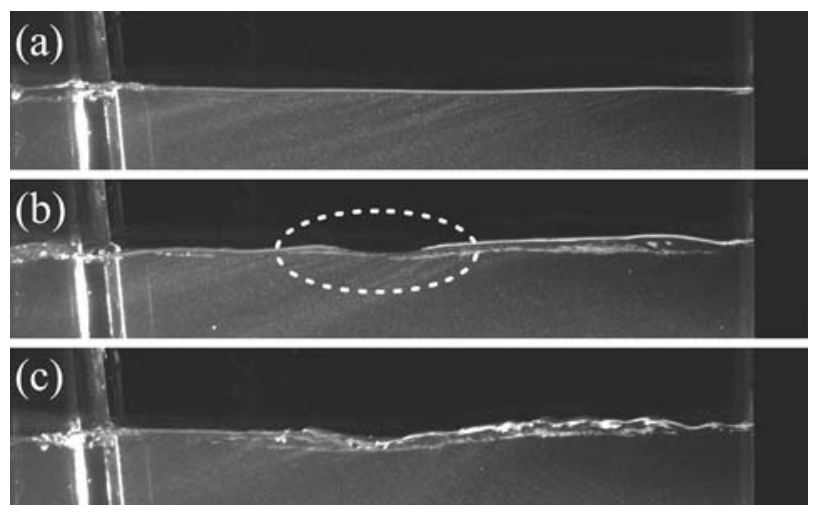

Fig. 4-Example meniscus images. (a) Well-detected image, (b) shadow, and $(c)$ diffuse reflections.

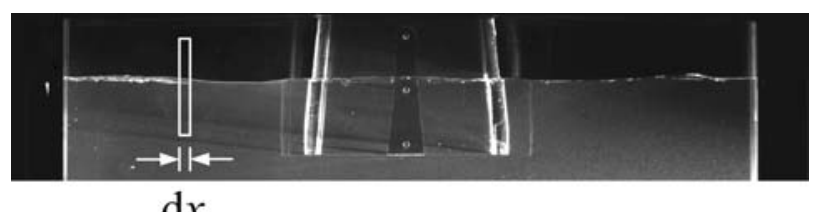

(a)

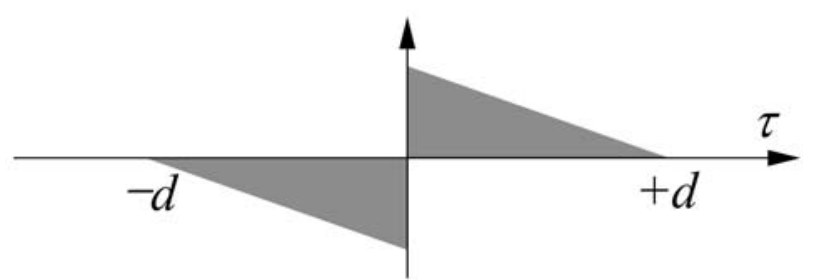

(b)

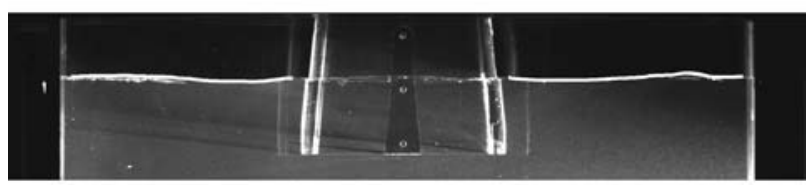

(c)

Fig. 5-Surface detection procedure. (a) PIV image $f(x, y)$, (b) convolution operator $\mathrm{c}(\tau)$, and $(c)$ detected meniscuses.
This approach is similar to the intensity gradient concept of Law et al. ${ }^{[19]}$ The present treatment had a smoothing effect on the $x$ and $y$ directions, respectively. The final step to obtain $y_{\text {meniscus }}$ was a Gaussian fitting with three points, which was used for subpixel accuracy. The locations of the meniscus were obtained successively as shown in Figure 5(c).

\section{RESULTS AND DISCUSSION}

\section{A. Time-Varying Meniscus Level}

The variations in time of the meniscus height in the $1.8 \mathrm{~T}$ - and $2.2 \mathrm{~T}$-funnel models under the maximum flow rate $\left(50 \mathrm{~m}^{3} / \mathrm{h}\right)$ are shown in Figures 6 and 7 , respectively. The meniscus height was obtained every 8 pixels in the horizontal direction, and the accuracy of the height was $0.38 \mathrm{~mm}$ (0.5 pixels). Weak meniscus fluctuations were observed in the $1.8 \mathrm{~T}$ system (Figure 6). In the $2.2 \mathrm{~T}$ system, however, strong fluctuations were observed during a period of $1.2 \sim 1.4$ seconds (Figure 7). These findings indicate that the ratio of mold thickness to mold width has a significant influence on the meniscus fluctuations, which is consistent with the results of Lawson and Davidson, ${ }^{[4]}$ who found that the meniscus oscillations in a parallel mold without a funnel

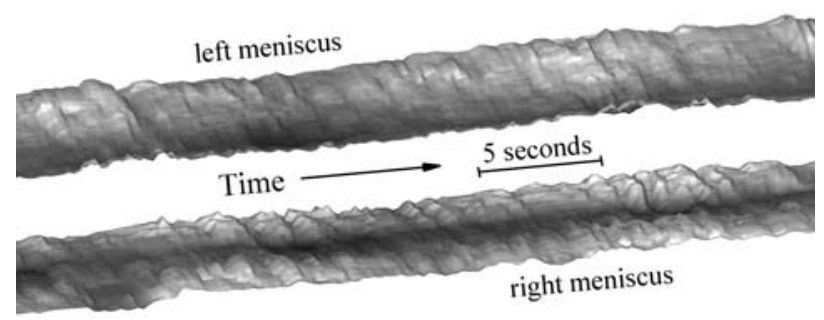

(a)

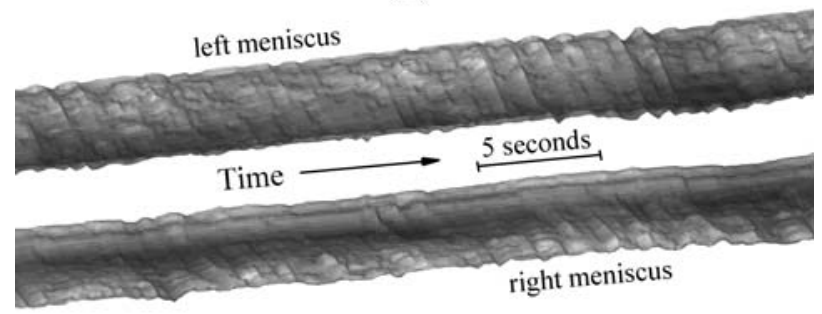

(b)

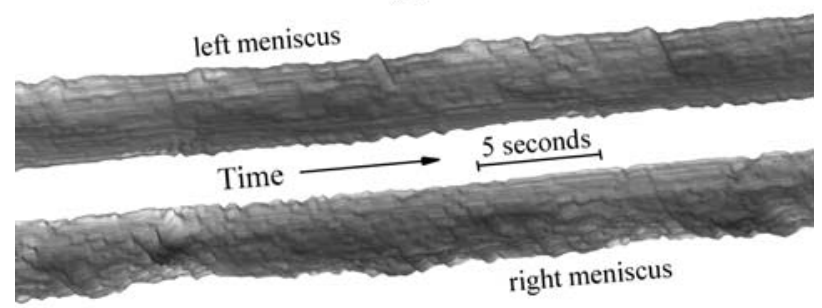

(c)

Fig. 6-Time-varying meniscus profiles for a funnel thickness of 1.8T. (a) Type I, (b) Type II, and (c) Type III. 


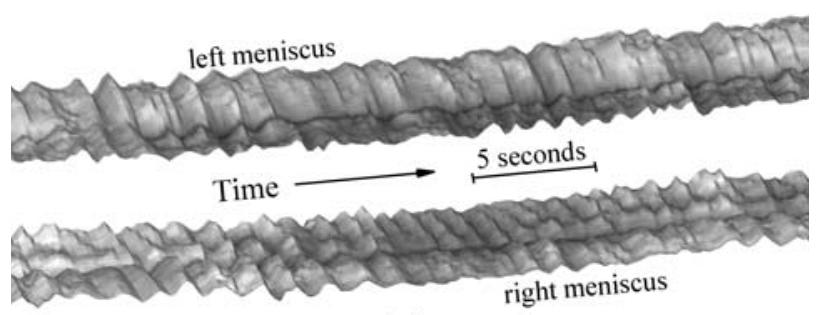

(a)

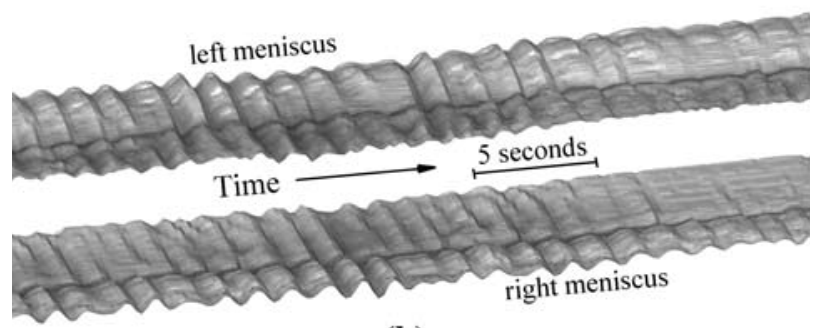

(b)

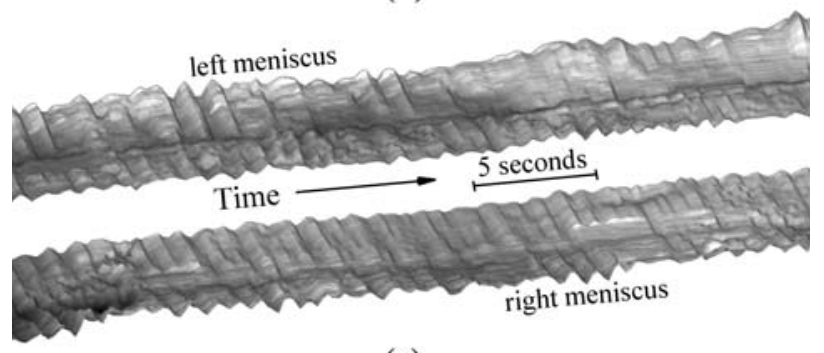

(c)

Fig. 7-Time-varying meniscus profiles for a funnel thickness of 2.2T. (a) Type I, (b) Type II, and (c) Type III.

changed from stable to unstable as the width-to-thickness ratio varied.

To find the periodic modes of the fluctuations, fast Fourier transforms (FFTs) were applied to the meniscus fluctuation data for the $1.8 \mathrm{~T}$ and $2.2 \mathrm{~T}$ systems (Figures 8 and 9, respectively). Because each data set consists of 225 PIV pairs, the FFT minimum resolution was $0.039 \mathrm{~Hz}$. The FFT results were normalized by the natural wave frequency, which was calculated by $\left(f_{0}=(\mathrm{g} / 4 \pi \mathrm{W})^{1 / 2}=0.760 \mathrm{~Hz}\right)$. Note that this formula does not include the mold thickness. For the thickness of $1.8 \mathrm{~T}$, peaks were observed at $f / f_{0}=1.028,1.028$, and 0.976 for the Type I, II, and III systems, respectively. Numerous blurred ridges were detected in other frequency ranges, but their strengths were not significant. For the thickness of 2.0T, peaks were observed at 1.052, 1.052 , and 1.028 for the Type I, II, and III systems, respectively.

The peak frequencies of the three $2.2 \mathrm{~T}$ systems were slightly higher than those of the corresponding $1.8 \mathrm{~T}$ systems. This result may be attributed to the fact that the SEN acted as a damper (i.e., the SEN has a blockage effect on the cross flow, which decreases the flow momentum). For all three SEN types, the clear peak was observed in the flat mold region $(x / W>0.3)$. Two or three ridges were observed for the Type III SEN. These periodic meniscus fluctuations were related directly to the self-sustained oscillations inside the mold.

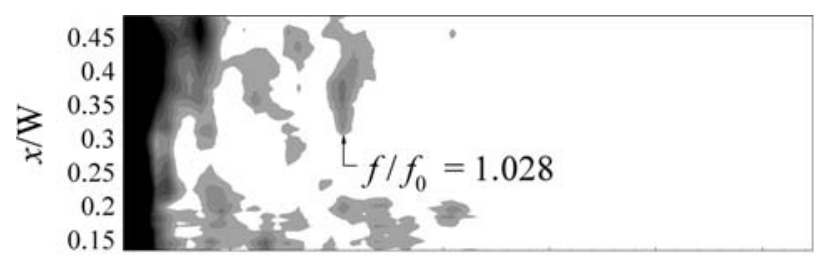

(a)

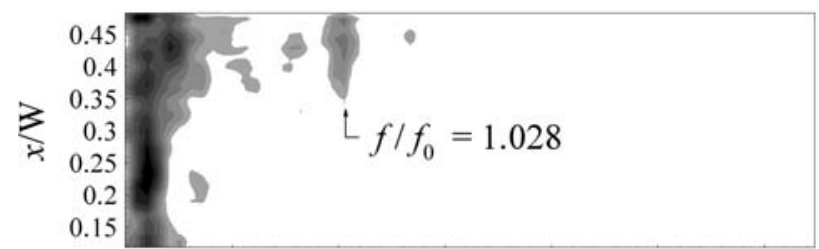

(b)

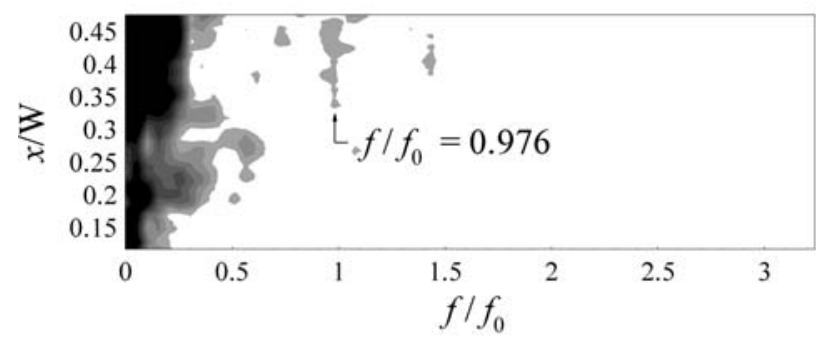

(c)

Fig. 8-FFT results for a funnel thickness of 1.8T. (a) Type I, (b) Type II, and (c) Type III.

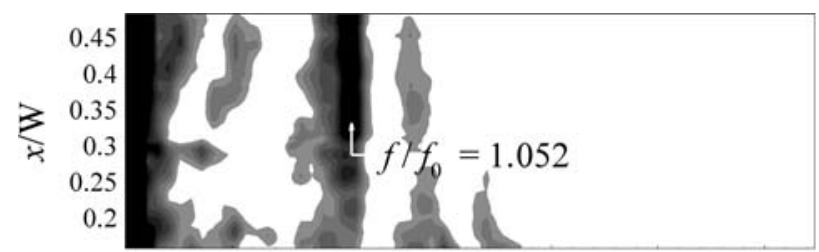

(a)

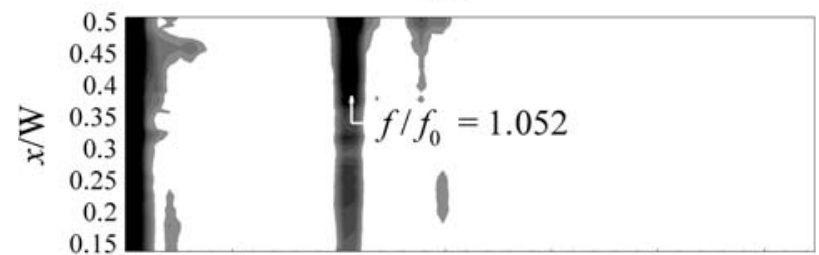

(b)

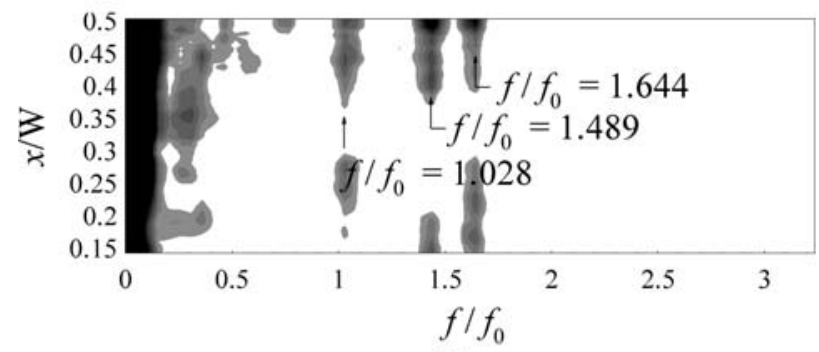

(c)

Fig. 9-FFT results for a funnel thickness of 2.2T. (a) Type I, (b) Type II, and (c) Type III.

Because these fluctuations disturb the formation of a solidification shell, unstable oscillations should be reduced in the real casting process. 


\section{B. Vortices on the Meniscus}

Vortex generation within a mold can cause defects in the final product. Several previous studies have examined this phenomenon. ${ }^{[5-7]}$ Recently, Torres-Alonso et $a l .{ }^{[8]}$ demonstrated vortex generation at the side of the SEN in a funnel mold by numerical simulation as well as in water-model tests. These phenomena originate from cross-flow separation induced by the shape of the SEN. To prevent vortex generation, in this study, the side of the SEN was treated as a streamlined shape. However, as shown in Figure 10, vortices still were generated in the gap between the SEN and the center of the funnel. It seems that the flow momentum of the cross flow was preserved without separation loss. As shown in Figure 11, two cross flows collided at the center of the funnel and rolled up toward the SEN, which is similar to the "bathtub" findings of Gebhard et al. ${ }^{[5]}$ Most vortices in the present study were generated in pairs. To see the effect of the extraction speed on the presence of vortices, the ratio of the number of vortex images to the total number of images is listed in Table I. In this investigation, only vortices with depths larger than $4.6 \mathrm{~mm}$ (6 pixels) were counted. Note that the numbers of vortices was reduced considerably for the Type III SEN compared with the other two types. Moreover, for the Type III nozzle, the vortices were observed in less than 10 pct of images for all extraction speeds. At low flow rates $(0.2 \sim 0.4 U)$, the Type I and II systems showed stable features; however, vortex generation rapidly increased in these systems when the flow rate was increased. The lower vortex formation in the Type III

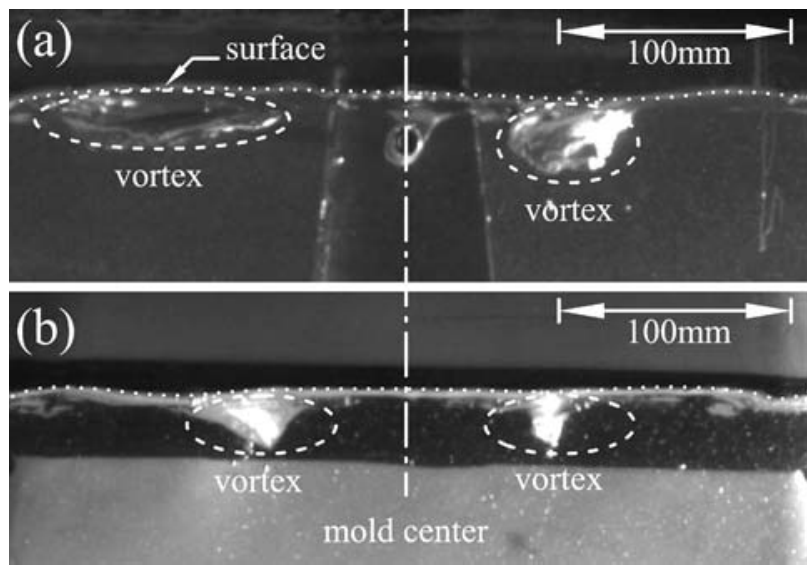

Fig. 10-Vortex image samples on the meniscus. (a) Type I and (b) Type II.

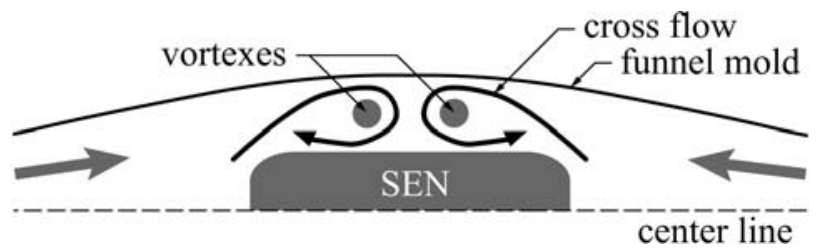

Fig.11-Schematic of the vortex generation.
SEN may be generated by the less periodic meniscus flows. As shown in Figures 6-9, the left and right meniscuses fluctuated in an opposite phase because of the natural oscillation, and these fluctuations generated a hydraulic head, which caused vortices. However, the meniscus fluctuations of the Type III SEN were less than those of other cases because of the small cross flow; the vortex generation then decreased.

\section{Mean Flows}

Velocity vectors and streamlines for the $1.8 T$ funnel are shown in Figure 12 for the three types of SEN. Three flow rates were chosen for comparison $(1.0 \mathrm{U}$, $0.6 U$, and $0.2 U$ ). For the Type I system, one large recirculation was generated because of the strong downward throughput from the SEN. Varying the flow rate had no significant effect on the streamline shapes, which means that the inner flow was not related to the flow rate, although the effect of the Reynolds number was significant. For the Type II nozzle, by contrast, the streamline shapes were affected greatly by the flow rate. When the flow rate was small $(0.2 U)$, small flow circulations were generated around the exit of the SEN. However, as the flow rate increased, the two recirculation regions merged into a single large flow rate. For flow rates higher than $0.5 \mathrm{U}$, the side throughputs were not strong enough to form an additional circulation; rather, the recirculation regions merged into a large recirculation at center. At a high flow rate, the streamline shapes of Type I were similar to those of Type II. Because the center of the recirculation flow of Type I was located closer to the meniscus $(y / \mathrm{W}=-0.15)$ than that of Type II $(y / \mathrm{W}=-0.10)$, the flow momentum delivered from the inner flow to the meniscus was higher. The system with a Type III nozzle showed complex flow patterns because of the five SEN exits. The flow patterns for the Type III SEN seemed to be similar regardless of the flow rate; however, the center location of the highest recirculation was $x / \mathrm{W}=0.25$ for $0.2 \mathrm{U}$ and $x / \mathrm{W}=0.33$ for $1.0 U$.

Figure 13 shows detailed velocity vector plots in the vicinity of the meniscus under the maximum flow rate $(1.0 U)$ for Types I, II, and III. Because the streamlines of Type I were similar to those of Type II, the velocity vectors of Type I showed a similar flow pattern to those of Type II. For the Type I system, the velocity magnitude from the meniscus to $y=0.1 \mathrm{~W}$ was similar to that of Type II. However, the Type III system showed a different flow pattern. The velocity magnitude was

Table I. Numbers of Vortex Events for a Funnel of Thickness $1.8 \mathrm{~T}$

\begin{tabular}{lrrr}
\hline Extraction Speed & Type I & Type II & Type III \\
\hline $0.2 U$ & $1.2 \mathrm{pct}$ & $2.0 \mathrm{pct}$ & $0.4 \mathrm{pct}$ \\
$0.4 U$ & $2.8 \mathrm{pct}$ & $1.6 \mathrm{pct}$ & $0.8 \mathrm{pct}$ \\
$0.6 U$ & $5.2 \mathrm{pct}$ & $6.4 \mathrm{pct}$ & $3.2 \mathrm{pct}$ \\
$0.8 U$ & $64.4 \mathrm{pct}$ & $62.0 \mathrm{pct}$ & $4.2 \mathrm{pct}$ \\
$1.0 U$ & $78.4 \mathrm{pct}$ & $81.6 \mathrm{pct}$ & $5.8 \mathrm{pct}$ \\
\hline
\end{tabular}



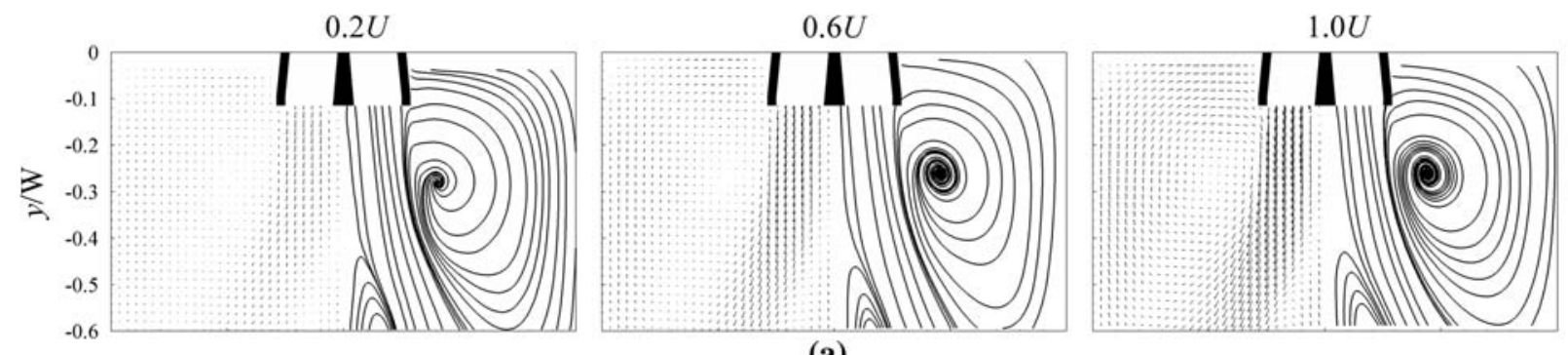

(a)
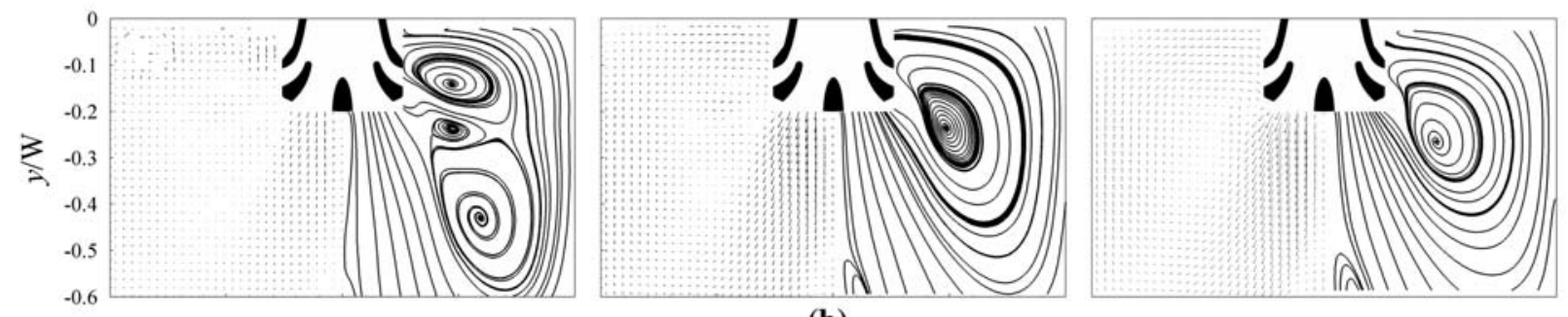

(b)
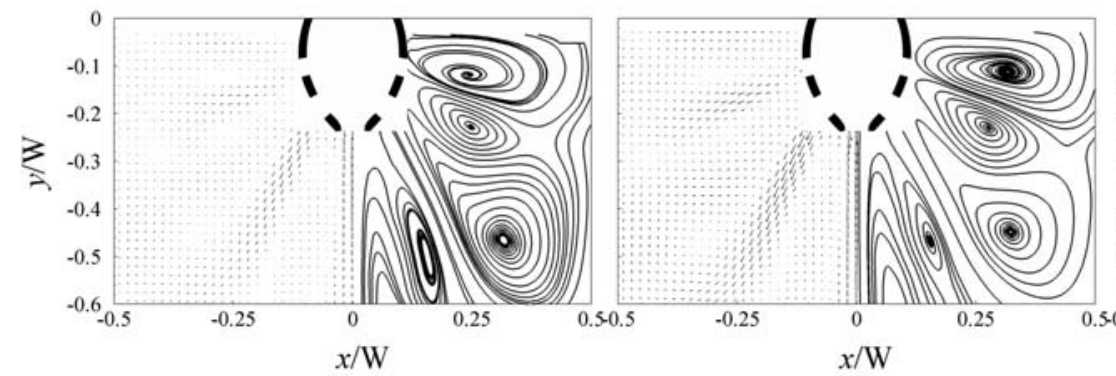

(c)

Fig. 12-Mean velocity vectors and streamlines. (a) Type I, (b) Type II, and (c) Type III.

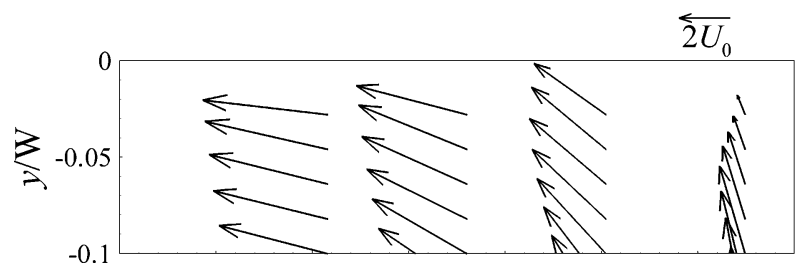

(a)

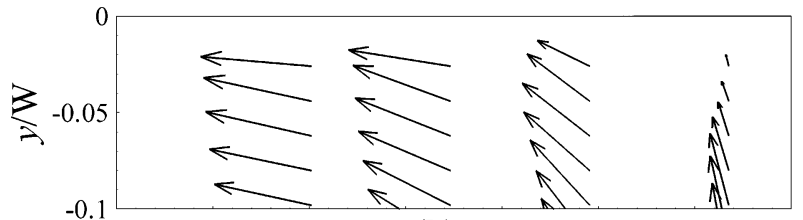

(b)

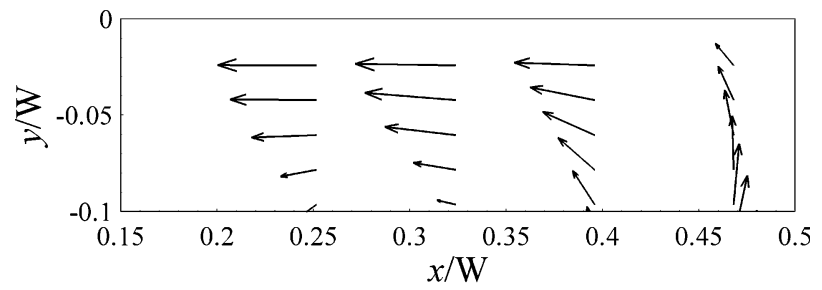

(c)

Fig. 13-Mean velocity vectors in the vicinity of the meniscus. (a) Type I, (b) Type II, and (c) Type III.

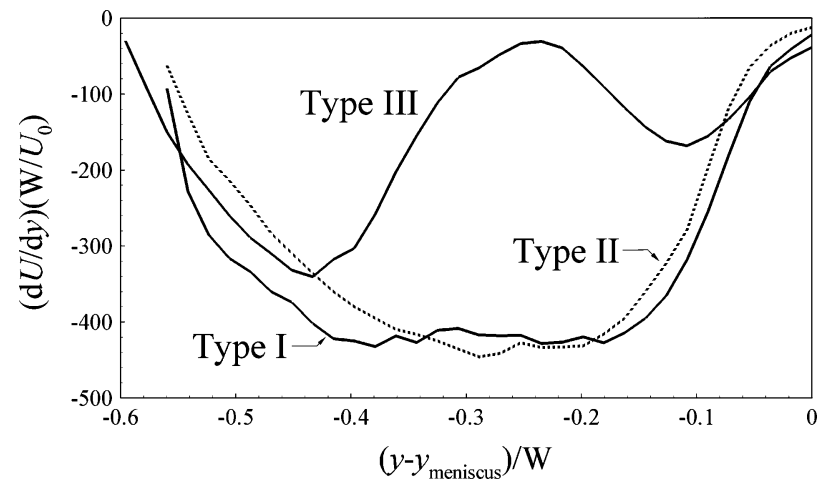

Fig. 14 - Shear stress at the right wall $(\mathrm{x} / \mathrm{W}=0.5)$.

smaller than in the Type I and II flows because of the small recirculation near the meniscus. The shear stress profiles at the right wall $(x / W=0.5)$ determined from the PIV results are shown in Figure 14. The maximum shear stresses for the Type I and II systems were about -430 , whereas the maximum shear stress for the Type III system was -340 . The shear stress profile for Type III had a wavy form. The momentum of the throughput at the SEN exit dissipated well for the Type III nozzle because of the many recirculation structures. 


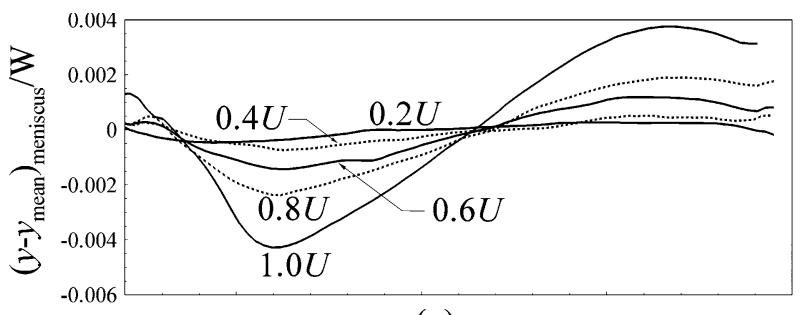

(a)

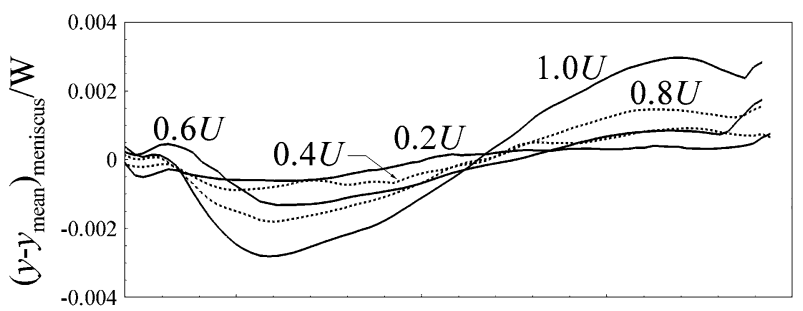

(b)

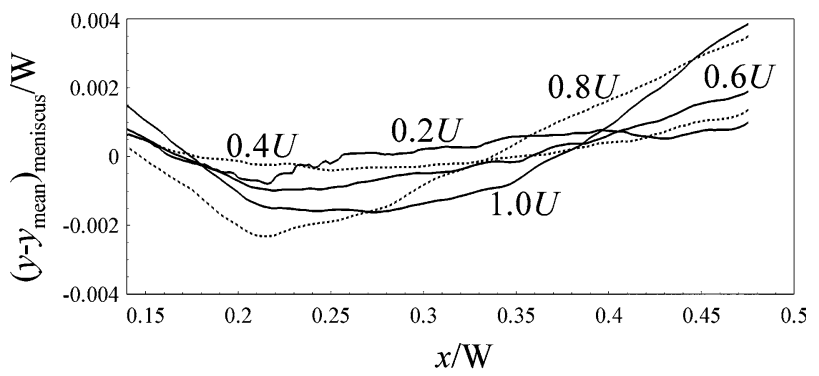

(c)

Fig. 15-Time-averaged meniscus level for a funnel thickness of 1.8T. (a) Type I, (b) Type II, and (c) Type III.

Accordingly, the shear stress-induced upward force on the solidification shell was decreased.

\section{Meniscus Levels}

Figure 15 shows the time-averaged meniscus levels at different flow rates for the three types of SEN. The global shape of Type I is similar to that of Type II. For both nozzle types, the average meniscus level was greatest at $x / \mathrm{W}=0.45$ and smallest at $x / \mathrm{W}=0.22$ at $1.0 \mathrm{U}$, which indicates that the inner flow structures of Type I are similar to those of Type II. However, the average meniscus levels of the Type III system were totally different from those of Types I and II. For the Type III system, a similar flow pattern was observed regardless of the flow rate (Figure 12); however, the data in Figure 15 demonstrate that the meniscus level varies depending on the flow rate because the relation between the meniscus and the flow rate was not weak. In addition, the meniscus level of the Type III system showed a V shape at most flow rates, which was consistent with the optimal bifurcated SEN design of Brummayer et al. ${ }^{[20]}$

The horizontal and vertical meniscus velocities $u$ and $v$ are shown in Figures 16 and 17, respectively. The horizontal meniscus velocity $u$ of Type I was similar to that of Type II because of the inner flow resemblance,

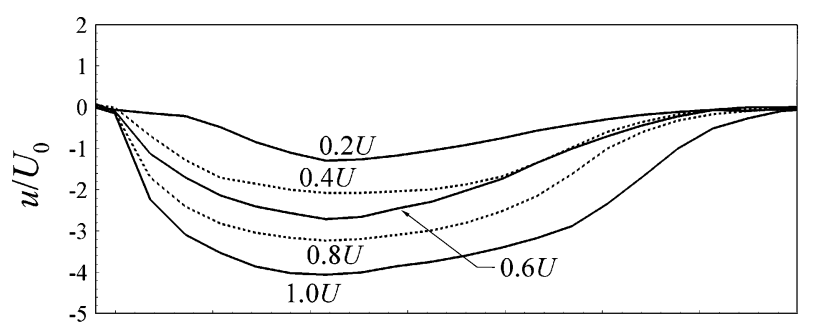

(a)

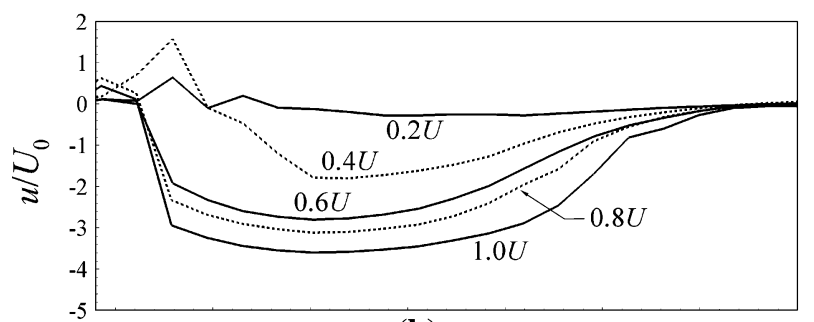

(b)

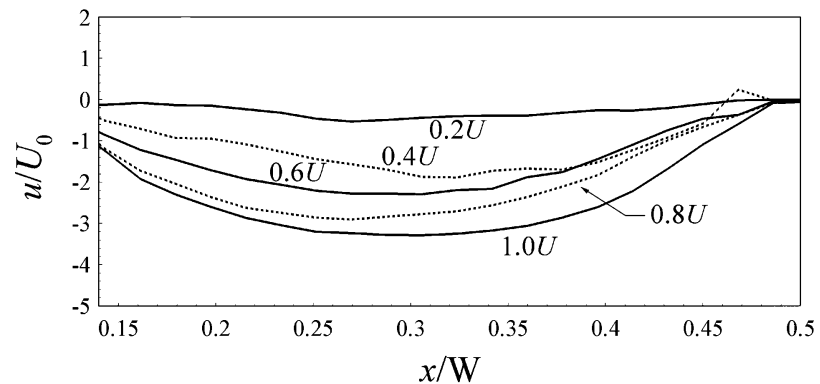

(c)

Fig. 16-Time-averaged horizontal meniscus velocity for a funnel thickness of 1.8T. (a) Type I, (b) Type II, and (c) Type III.

although the systems show dissimilarities at flow rates of $0.4 U$ and $0.2 U$. For Types I and II, an upward flow near the wall with large $v$ values were observed. The magnitude of $u$ at the center was large because of the inner recirculation influence. For the Type III systems, however, $v$ was small. In sum, the meniscus levels depended on the meniscus velocity, and the flow recirculation inside the mold had a significant influence on the meniscus.

\section{CONCLUSIONS}

We performed PIV measurements in a funnel-type water model to elucidate the quantitative characteristics of the flow oscillations inside the mold and the meniscus fluctuations. A full-size water model was constructed, and PIV measurements were recorded across the entire flow within the model. Three SEN designs were tested with two funnel models under five flow rate conditions. To improve the accuracy of the results, the window deformation and IPX techniques were employed. An edge-detection algorithm was used to find the meniscus location. We found that the ratio of mold thickness to mold width influences the meniscus fluctuations and that the strength of the self-sustained oscillations is related closely to the funnel size. The peak frequency of the 


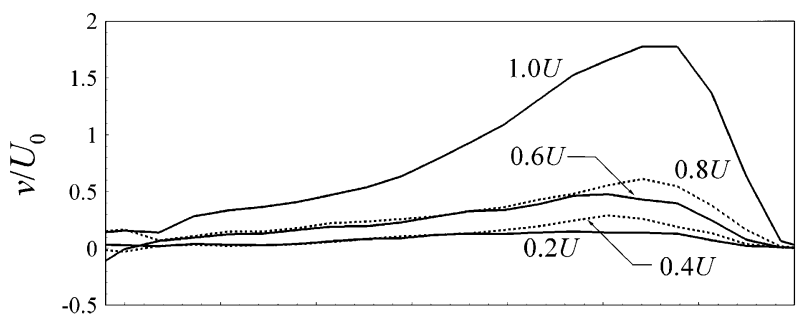

(a)

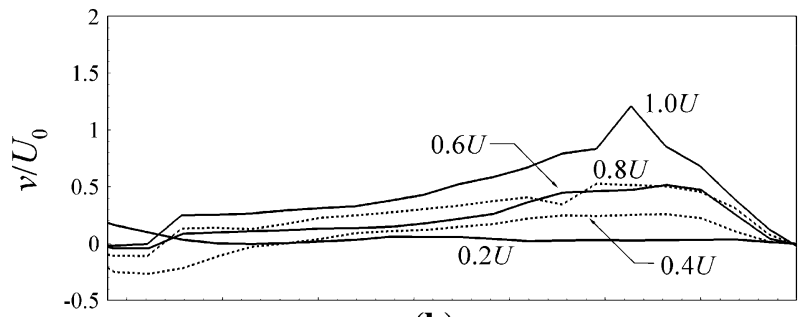

(b)

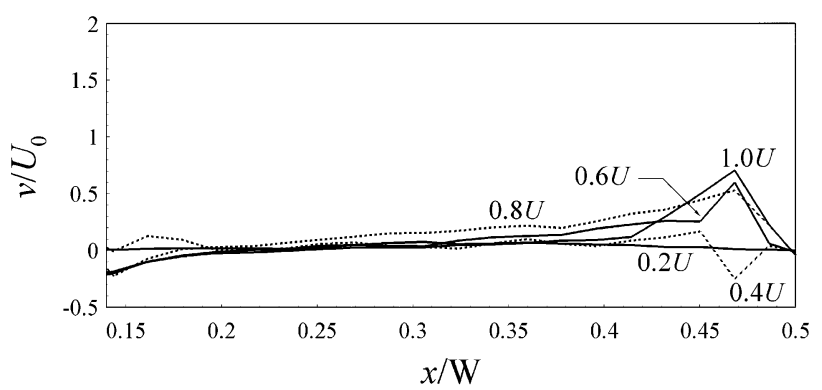

(c)

Fig. 17-Time-averaged vertical meniscus velocity for a funnel thickness of 1.8T. (a) Type I, (b) Type II, and (c) Type III.

Type I system $(0.82 \mathrm{~Hz})$ is slightly higher than that of the Type II system $(0.78 \mathrm{~Hz})$. The clear peak is distributed in the flat mold region $(x / \mathrm{W}>0.3)$. The periodic meniscus fluctuations are related directly to the self-sustained oscillations inside the mold. As the ratio of funnel size to width decreases, the periodic features decrease. At low flow rates $(0.2 \sim 0.4 U)$, Type I and II systems show stable features. For Type I, one large recirculation is generated because of the strong downward throughput from the SEN. Varying the flow rate has no significant effect on the streamline shapes in the Type I system. For Type II, by contrast, the streamline shapes show a strong dependence on the flow rate. When the flow rate is small $(0.2 U)$, small flow circulations are generated around the exit of the SEN. However, as the flow rate increases, two recirculation regions merge into a single large region. At flow rates higher than $0.5 \mathrm{U}$, the side-throughputs are not strong enough to form an additional circulation; rather, the recirculations merge into a single recirculation at the center. At high flow rates, the streamline shapes of Type I are similar to those of Type II. The Type III system shows complex flow patterns because of the five SEN exits. Similar flow patterns were observed at all flow rates. However, as the flow rate increases, vortex generation rapidly increases. The lower vortex in the Type III flow may be generated by the periodic meniscus flows. The vortex pairs appear between the SEN and the funnel and are generated by the crush and the rolling of the cross-flow. The formation of these vortices is affected by the meniscus velocity as well as the velocity in the vicinity of the meniscus. As the number of recirculation regions increases, the quantity of the flow that influences the meniscus is reduced. As a result, the inner and meniscus flows are stabilized.

\section{NOMENCLATURE}

$\mathrm{W}$
$\mathrm{T}$
$\mathrm{L}$
$d_{\mathrm{i}}$
$V_{\mathrm{i}}$
$U$
$U_{0}$
$d t$
$w$
$(x, y)$
$\left(x_{0}, y_{0}\right)$
$f_{1}(x, y)$
$f_{2}(x, y)$
$f_{0}$
$u$
$v$

Mold width (mm)

Mold thickness (mm)

Mold length ( $\mathrm{mm})$

Inner diameter at the entrance of the SEN $(\mathrm{mm})$

Mean velocity at the entrance of the SEN $(\mathrm{m} / \mathrm{s})$

Maximum extraction speed $(\mathrm{m} / \mathrm{sec})$

Normalized extraction speed $(\mathrm{m} / \mathrm{sec})$

Time interval between successive laser exposures (ms)

Size of interrogation window (pixel)

Cartesian coordinate of the recorded image (pixel)

Center location of the first interrogation window (pixel)

First intensity field of a PIV image pair Second intensity field of a PIV image pair Natural wave frequency $(\mathrm{Hz})$

Velocity magnitude in $x$-direction $(\mathrm{m} / \mathrm{sec})$ Velocity magnitude in $y$-direction $(\mathrm{m} / \mathrm{sec})$

\section{REFERENCES}

1. T. Honeyands and J. Herbertson: Steel Res., 1995, vol. 66 (7), pp. 287-93.

2. B.M. Gebert, M.R. Davidson, and M.J. Rudman: Appl. Math. Model., 1998, vol. 22, pp. 843-50.

3. D. Gupta and A.K. Lahiri: Metall. Mater. Trans. B, 1996, vol. 27, pp. 757-64.

4. N.J. Lawson and M.R. Davidson: J. Fluids Struct., 2001, vol. 15, pp. $59-81$.

5. M. Gebhard, Q.L. He, and J. Herbertson: 76th Steelmaking Conf. Proc., 1993 pp. 441-46.

6. B. Li, T. Okane, and T. Umeda: Metall. Mater. Trans. B, 2001, vol. 32, pp. 1053-66.

7. B. Li and F. Tsukihashi: ISIJ Int., 2005, vol. 45 (1), pp. 30-36.

8. E. Torres-Alonso, R.D. Morales, L.G. Demedices, A. Najera, J. Palafox-Ramos, and P. Ramirez-Lopez: ISIJ Int., 2007, vol. 47 (5), pp. 679-88.

9. J.Y. Lamant, M. Larrecq, A. Mouchete, Y. Codur, J. Gancarz, and A. Leclercq: 6th Int. Iron and Steel Congress, 1999, pp. 317-24.

10. M. Iguchi, J. Yoshida, T. Shimizu, and Y. Mizuno: ISIJ Int., 2000, vol. 40 (7), pp. 685-91.

11. H. Nam, H.W. Park, and J.K. Yoon: ISIJ Int., 2000, vol. 40 (9), pp. 886-92.

12. M. Raffel, C.E. Willert, S.T. Wereley, and J. Kompenhans: Particle Image Velocimetry: A Practical Guide, $2^{\text {nd }}$ ed., New York, NY: Springer.

13. S. Ashforth-Frost, B.N. Dobbins, K. Jambunathan, X. Wu, and X.Y. Ju: (1993) Optical Diagnostics in Fluid and Thermal Flow, P. Buchhave, L. Lading, and G. Wigley, eds., Springer, New York, NY, 1993. 
14. H.T. Huang, H.E. Fiedler, and J.J. Wang: Exp. Fluids, 1993, vol. 15 , pp. $263-73$.

15. K. Jambunathan, X.Y. Ju, B.N. Dobbins, and S. Ashforth-Frost: Meas. Sci. Technol., 1995, vol. 6 (5), pp. 507-14.

16. B.J. Kim and H.J. Sung: Exp. Fluids, 2006, vol. 41 (3), pp. 499-511.

17. L. Tsuei and Ö. Savaş: Exp. Fluids, 2000, vol. 29, pp. 203-14.
18. F. Scarano: Meas. Sci. Technol., 2002, vol. 13, pp. R1-19.

19. C.N.S. Law, B.C. Khoo, and T.C. Chew: Exp. Fluids, 1999, vol. 27 (4), pp. 321-31.

20. M. Brummayer, P. Gittler, and J. Watzinger: Second Int. Conf. on Computational Fluid Dynamics in the Minerals and Process Industries, 1999 pp. 217-22. 